\title{
ELECTROLYTE DISTURBANCE IN HEMORRHAGIC AND NON-HEMORRHAGIC STROKE PATIENTS IN AL-DIWANIYAH TEACHING HOSPITAL
}

\author{
AQEEL RAHEEM HASSAN ${ }^{1 *}$, ZAHRAA ADEL ARYAN ${ }^{2}$ \\ 1Department of Internal Medicine, College of Medicine, University of AL-Qadisiyah, Diwaniyah, Iraq.2Department of Al-Dewaniyah \\ Teaching Hospital, Al-Dewaniyah Province, Iraq.Email: aqeel.r.hassan@gmail.com
}

Received: 18 August 2018, Revised and Accepted: 24 September 2018

\section{ABSTRACT}

Objectives: Although there are many studies on stroke, few studies on electrolyte disturbance have been done in our country, even on the outside. Our aim in this study is to estimate the level of serum potassium and sodium in acute stroke patients with comparison to patients of the control group.

Methods: Our study is a comparative cross-sectional study conducted on patients in the neurological center who suffer from stroke and others in medicine department admitted for any disease other than cerebrovascular accidents (CVA) they consider the control group. All were in AL-Diwaniyah Teaching Hospital from April to July 2018. The level of potassium and sodium from all patients is estimated. Patients with ischemic stroke, hemorrhagic stroke, and transient ischemic attack (TIA) were classified into having Glasgow coma scores (GCS) of 3-8, 9-12, and 13-15, respectively.

Result: Significant difference was seen in the distribution of patients according to GCS levels ( $p=0.014$ ). Mean serum sodium was significantly lowest in hemorrhagic stroke, then ischemic stroke, followed by TIA, and the highest sodium level was seen in the control group (p<0.001). Mean serum potassium was significantly lowest in hemorrhagic stroke, then ischemic stroke, followed by TIA, and the highest sodium level was seen in the control group ( $\mathrm{p}<0.001)$. Mean serum-to-potassium ratio was significantly highest in hemorrhagic stroke, then TIA, followed by ischemic stroke, and finally, by control group $(\mathrm{p}<0.001)$.

Conclusion: This study reveals that, in hemorrhagic stroke, the incidence of electrolytes imbalance was more than ischemic and which was mostly hyponatremia and hypokalemia.

Keyword: Stroke, Electrolyte Disturbance, Sodium, Potassium.

(c) 2018 The Authors. Published by Innovare Academic Sciences Pvt Ltd. This is an open access article under the CC BY license (http://creativecommons. org/licenses/by/4. 0/) DOI: http://dx.doi.org/10.22159/ajpcr.2018.v12i1.29173

\section{INTRODUCTION}

Cerebrovascular accidents (CVAs) are a main public health problem. It is well distributed in the whole world and is asserted to be the second top cause of death in the world. Stroke causes significant impairments particularly in the older age group and is among the major health matters in several countries [1]

Electrolyte disturbance is commonly found in acute stroke events Recently research with electrolyte disturbances focusing on risk factors (RFs) of stroke, its prevalence, and association with other medical condition, not only on the neuroendocrine mechanism. Many stroke patients die either due to the primary disease or due to its consequences. Management of stroke patient aimed not only on the treatment of the primary disease but also on the avoidance of severe consequences of stroke including dyselectrolytemia, aspiration pneumonia, malnutrition, pulmonary embolism, DVT, bowel or bladder dysfunction, urinary tract infection, contractures, skin breakdown, and joint abnormalities. An early and accurate forecasting of stroke outcome in the emergency department is pivotal for decision-making, as well as in assessing patient's prognosis.

The reports on the association between electrolyte imbalance and severity of acute stroke are still in limited number, although there are some data about a large number of electrolyte disturbances in acute stroke events. There is a deficiency of data about this association, especially from the developing countries. Electrolyte disturbances are usually present in acute stroke setting as hypokalemia or hyponatremia which is the most common type of disturbance. Electrolyte disturbances such as hyponatremia resulting from either the syndrome of inappropriate antidiuretic hormone secretion, inappropriate fluid intake and loss, or high level of brain natriuretic peptides (BNP) can result in consequences such as seizures or death.
The most common presenting symptoms of patients with hemorrhagic stroke are a headache and vomiting. Vomiting is also an important factor of electrolyte disturbance [2]. Published reports about stroke suggest that CVD occurs with increasing frequency at all ages and in both the sexes. Prospective studies on acute stroke found that hypertension, diabetes mellitus, dyslipidemia, obesity, smoking, and family history are important RFs [3-5]. The objectives of this study reported here are to identify the common electrolyte disturbance in the acute phase of multiple types of stroke patients and their relation with some usual clinical presentation and outcome.

\section{METHODS}

It is a comparative cross-sectional study conducted on patients admitted to neurological center from April to July 2018 in comparison to patients admitted to medicine department for any cause other than CVA in the same hospital and at the same period.

\section{Inclusion criteria}

Patients of either sex $>40$ years of age with acute stroke admitted within $48 \mathrm{~h}$ of onset and fulfilling the WHO definition of stroke and confirmation of stroke with CT scan of the brain, patients with no history of endocrine or kidney diseases, patients who were not in resuscitation phase, and patients who are not in diuretic therapy were included in the study.

\section{Exclusion criteria}

Any patients who have complication that could affect electrolyte level, patients who have any neurological deficit secondary to head injuries, intracerebral hemorrhage (ICH), subdural hemorrhage, epidural hemorrhage, or an infarction which is caused by an infection/tumor (space occupying lesion), etc., and also patients 
who have preexisting severe physical or cognitive disabilities were excluded from the study.

\section{Patient consent}

The authors certify that they have obtained all appropriate patient consent forms. In the form, the patient(s) has/have given his/her/their consent for his/her/their images and other clinical information to be reported in the journal. The patients understand that their names and initials will not be published and due efforts will be made to conceal their identity.

\section{Statistical analysis}

Data were collected, summarized, analyzed, and presented using two software programs; these were the Statistical Package for the Social Sciences version 23 and Microsoft Office Excel 2013. Numeric variables were presented as mean, standard deviation, and range, whereas categorical variables were expressed as number and percentage. Association between categorical variables was assessed using either Chi-square test or Yates correction for continuity when $>20 \%$ of cells have expected counts $<5$. Comparison of mean values between the two groups was performed using one-way analysis of variance. The level of significance was considered at $\mathrm{p} \leq 0.05$.

\section{RESULTS}

Diabetes mellitus was seen in 10 (17.5\%), 3 (23.1\%), and 4 (33.3) patients with ischemic stroke, hemorrhagic stroke, and transient ischemic attack (TIA) patients, respectively, and the difference statistically was not significant $(\mathrm{p}=0.459)$. Hypertension was seen in $31(54.4 \%), 6(46.2 \%)$, and 7 (58.3\%) patients with ischemic stroke, hemorrhagic stroke, and TIA patients, respectively, and the difference statistically was not significant $(\mathrm{p}=0.814)$. Smoking was seen in $33(57.9 \%), 8(61.5 \%)$, and $7(58.3 \%)$ patients with ischemic stroke, hemorrhagic stroke, and TIA patients, respectively, and the difference statistically was not significant $(\mathrm{p}=0.971)$. Dyslipidemia was seen in $34(59.6 \%), 7(53.8 \%)$, and 7 (58.3\%) patients with ischemic stroke, hemorrhagic stroke, and TIA patients, respectively, and the difference statistically was not significant $(\mathrm{p}=0.929)$. Overweight or obesity was seen in 19 (33.3\%), 5 (38.5\%), and 4 (33.3\%) patients with ischemic stroke, hemorrhagic stroke, and TIA patients, respectively, and the difference statistically was not significant $(\mathrm{p}=0.938)$. Family history was seen in $20(35.1 \%), 4(30.8 \%)$, and $5(41.7 \%)$ patients with ischemic stroke, hemorrhagic stroke, and TIA patients, respectively, and the difference statistically was not significant $(\mathrm{p}=0.848)$. Patients with ischemic stroke were classified into 9 (15.8\%), 34 (59.6\%), and $14(24.6 \%)$ having Glasgow coma scores (GCS) of 3-8, 9-12, and 13-15, respectively. Patients with hemorrhagic stroke were classified into $5(38.5 \%), 7(53.8 \%)$, and $1(7.7 \%)$ having GCS of 3-8, 9-12, and $13-15$, respectively. Patients with TIA were classified into $0(0.0 \%)$, $5(41.7 \%)$, and $7(58.3 \%)$ having GCS of $3-8,9-12$, and $13-15$, respectively. A significant difference was seen in the distribution of patients according to GCS levels $(p=0.014)$. Mean serum sodium was significantly lowest in hemorrhagic stroke, then ischemic stroke, followed by TIA, and the highest sodium level was seen in the control group $(\mathrm{p}<0.001)$. The mean of serum potassium was significantly lowest in hemorrhagic stroke, then ischemic stroke, followed by TIA, and the highest sodium level was seen in the control group $(\mathrm{p}<0.001)$. Mean serum sodium-to-potassium ratio was significantly highest in hemorrhagic stroke, then TIA, followed by ischemic stroke, and finally, by control group $(\mathrm{p}<0.001)$. The rate of hyponatremia was $2(2.2)$, 20 (35.1), 5 (38.5), and 2 (16.7), in control, ischemic, hemorrhagic, and TIA groups, respectively. The rate of hypokalemia was 1 (1.1), 4 (7.0), 2 (15.4), and 0 (0.0) in control, ischemic, hemorrhagic, and TIA groups, respectively (Table 1 ).

\section{DISCUSSION}

In this present study and according to imaging studies, there are 57 patients with ischemic stroke, 13 with ICH, and 12 with TIA in comparison to 90 patients in the control group.
Table 1: Correlation between serum electrolytes (serum sodium and potassium) and clinical characteristics

\begin{tabular}{|c|c|c|c|c|c|c|}
\hline \multirow[t]{2}{*}{ Characteristic } & \multicolumn{2}{|c|}{ Serum Na } & \multicolumn{2}{|c|}{ Serum K } & \multicolumn{2}{|c|}{$\mathrm{Na} / \mathrm{K}$ ratio } \\
\hline & $\mathbf{r}$ & $\mathbf{p}$ & $\mathbf{r}$ & $\mathbf{p}$ & $\mathbf{r}$ & $\mathbf{p}$ \\
\hline Stroke type & 0.206 & 0.064 & -0.055 & 0.625 & 0.150 & 0.180 \\
\hline Age & -0.197 & 0.077 & -0.328 & $0.003^{*}$ & 0.301 & $0.006^{*}$ \\
\hline Gender & -0.144 & 0.196 & -0.214 & 0.053 & 0.110 & 0.326 \\
\hline Diabetes & 0.170 & 0.126 & -0.045 & 0.688 & 0.099 & 0.377 \\
\hline Hypertension & 0.234 & $0.035^{*}$ & 0.215 & 0.053 & -0.131 & 0.241 \\
\hline Smoking & -0.085 & 0.448 & -0.026 & 0.818 & -0.002 & 0.986 \\
\hline Dyslipidemia & -0.012 & 0.918 & -0.087 & 0.435 & 0.067 & 0.548 \\
\hline $\begin{array}{l}\text { Overweight or } \\
\text { obesity }\end{array}$ & -0.150 & 0.180 & -0.088 & 0.432 & 0.030 & 0.789 \\
\hline Family history & 0.092 & 0.409 & 0.103 & 0.356 & -0.064 & 0.570 \\
\hline Residency & 0.010 & 0.929 & 0.016 & 0.885 & -0.019 & 0.865 \\
\hline Occupation & -0.049 & 0.659 & 0.095 & 0.396 & -0.104 & 0.352 \\
\hline Glasgow coma scale & 0.148 & 0.186 & 0.236 & $0.033^{*}$ & -0.181 & 0.104 \\
\hline
\end{tabular}

The current study shows that hemorrhagic stroke most commonly occurs at the older age patients (significant difference with the rest of patients and control subject $\mathrm{p}<0.001$ ), while ischemic and TIA mainly occur at middle age group and there was no significant difference in mean age with the control subject $(p>0.05)$.

According to a study in Manado General Hospital in Indonesia, they found that stroke is more commonly occurred in the middle age group and above also more in male than female. In this study, we found that there is no significant difference between male and female among the control and study groups $(p=0.952)$. In a community-based crosssectional study in Bao'an district, Shenzhen, China [6,7], there is more predominance of stroke in males than between females. The same results have been found in Saudi Arabia [8], and other countries, such as in German [9], the United Kingdom [10], the US [11], Italy [12], and Spain [13]. The multivariable studies indicated that sex was a significant factor in stroke, and men have 2 times more risk to develop a stroke than women.

Our study revealed that residency is not a RF for CVA because no significant difference was encountered among all stroke patients $(p=0.588)$. Furthermore, in this study, there is no significant difference according to the distribution of patients regarding their occupation $(p=0.588)$.

The RFs of stroke in the study group have the same effect for all types of stroke because the difference in distribution among ischemic, hemorrhagic, and TIA was statistically not significant.

The level of consciousness assessment for patients included in the present study was performed according to the GCS.

Our study shows a significant difference in the distribution of patients according to GCS levels (as mild, moderate, and severe disturbances of consciousness) $(\mathrm{p}=0.014)$. We detect that patients presenting with hemorrhagic stroke had a conscious level (median was 9) in comparative with ischemic and TIA (median 11 and 13), respectively. These findings may help to determine the stroke type because subarachnoid hemorrhage (SAH) is usually associated with significant increase in intracranial pressure and lowering in cerebral perfusion pressure this causing intermittent reduction of cerebral blood flow; thus, the main presenting symptoms of SAH is a loss of consciousness that mean sever GCS.

Distinguishing between ischemic and hemorrhagic stroke not depends on symptoms alone which are not enough specific. Any way generalized symptoms, such as nausea, vomiting, and headache, in addition to the altered level of consciousness, may give a hint to increasing intracranial 
pressure and usually found more in hemorrhagic strokes and large ischemic strokes.

Disturbance of the normal values of sodium and potassium will affect the fluid and electrolyte balance and may result in disturbing the normal physiological function and the neurotransmission of the brain in the form of collection of fluid and exchange of nutrients such as lactic acid and arachidonic acid which have mean role in the development of neurological signs and symptoms. The damage that results due to these consequences will result either in decreased cerebral blood flow or rupture of small vessels due to pressure imbalance. A study by Alam et al. [14] revealed that hyponatremia is the main electrolyte disturbances in CVA, both in ischemic and hemorrhagic stroke. Another study by Kusuda et al., [15] also showed hyponatremia, hypernatremia, hypokalemia, and hyperkalemia in CVA. The study also stated that hypernatremia is more common in hemorrhagic stroke and $57 \%$ of patients with hypernatremia in his study end by dead within 1 month of hospital admission indicating the importance of electrolyte disturbances in these patients.

In our study, we found that the hyponatremia and hypokalemia are more common in hemorrhagic stroke, followed by ischemic stroke, then TIA, and the highest level in the control group. Low sodium level was mainly detected among patients with hemorrhagic stroke followed by ischemic stroke patients, and there is a statistically significant association between hyponatremia and type of stroke. Many studies and researchers find that hyponatremia in acute CVA patient may affect the prognosis and severity of stroke negatively. Hypokalemia was more common among hemorrhagic stroke patients followed by ischemic stroke patients. In the study of Qureshi et al., it was detected that hyponatremia is most common than hypernatremia (30\% vs. $19 \%$ ) after aneurysmal SAH [16]. Hypernatremia was significantly associated with the worse prognosis, and there is a positive relationship between elevated serum sodium levels and poor GCS within 3 months following the ictus. Chandy et al. showed that hyponatremia was associated with a high risk of cerebral vasospasm after aneurysmal SAH [17]

A retrospective study by Sherlock et al. mentioned that 316 patients with aneurysmal SAH and hyponatremia were a common electrolyte imbalance between those patients (56\% overall prevalence) which result in longer hospital admission but without any effect on the mortality rate [18]. They detect that, in a major group of the hyponatremic patients, $(21.4-31.8 \%$, according to the treatment modality), this may develop within 7 days after SAH.

Mc Girt et al. revealed that an increase in the serum levels of the cerebral natriuretic peptide (BNP) was independently associated with hyponatremia, and the occurrence of hyponatremia rises significantly within $24 \mathrm{~h}$ after the onset of delayed ischemic neurologic deficits [19]. Hypokalemia occurs in hemorrhagic stroke patients more than ischemic stroke patients in comparison to control group.

Electrolyte disturbance has an effect on clinical characteristic revealed that there is a negative relationship between age and serum potassium; when age increases, serum potassium decrease; this may be the result of multiple causes. The normal physiological changes related to the aging affect the potassium normal values in the body. Furthermore, when the function of the kidneys decrease, this will effect on the urine output and cause a disturbance in the mechanisms that maintain reabsorption and excretion of nutrients. This high level of potassium will be execrated in urine. While at the level of the gastrointestinal system, vomiting and diarrhea can affect the proper absorption of dietary potassium. Other medical problems such as Crohn's or Cushing's disease, leukemia, or magnesium deficiency cause large loss of potassium. Some drugs such as the diuretics, insulin, steroid, laxatives, antibiotics, and theophyllines may change the normal absorption of potassium. Notably, when there is an increase in the levels of body sodium, blood pressure will increase. Renin-angiotensin system is affected by high salt diet. Damage to endothelial had a significant role in the effect of high sodium intake on blood pressure, although the exact mechanisms remain unknown. High blood pressure represents the major factor in the cardiovascular disease (such as stroke, cardiac failure, and coronary artery disease).

Potassium concentration through the membrane of the cell has a significant effect on the membrane potential so that when there is abnormal potassium level, this may affect membrane potential in neuronal, cardiac, and vascular tissues. A study by Cheng et al. showed that low potassium level can reduce the conductancehyperpolarization in potassium channel of skeleta muscle tissue. Severe muscle dysfunction, palpitations, cardiac dysrhythmias, and abnormality in neurological function may occur if there are few disturbances in potassium level [20]. Potassium intake can lower the stroke risk and this is shown by multiple meta-analyses studies, and the suggested mechanism could be that $\mathrm{K}^{+}$decreases the formation of free radicals and increases endothelial dysfunction. Gariballa et al. showed that low potassium level at admission may result in a 3-month mortality rate of acute ischemic stroke [21], while a study from Fofi et al. revealed that the relationship between mortality and the serum potassium level was not statistically significant [22-24]. These differences may be due to the discrepancy between the two studies in the population features. The study by Fofi et al. was focused on AIS patients with an onset-to- treatment of $<6 \mathrm{~h}$, while Gariballa et al. included patients with multiple types of stroke. Other groups revealed that $\mathrm{K}^{+}$can suppress vascular smooth muscle cel proliferation. A study performed at the first affiliated hospital of Xi'an Jiaotong University, Department of Neurology, revealed that serum potassium level $<3.7 \mathrm{mmol} / \mathrm{l}$ on admission is an indicator of poor outcome for 3 months after stroke following acute ischemic stroke event. Thus, hypokalemia is a significant factor in the worse outcome. Hence, acute ischemic stroke patients should be monitored because they are at higher risk of a poor prognosis by the effect of the serum potassium level at the time of admission. These findings will help physicians who usually monitor serum potassium with acute ischemic stroke and assess its level above $3.7 \mathrm{mmol} / \mathrm{l}$. Because $\mathrm{Mg} 2+$ can improve the $\mathrm{Na}+\mathrm{K}+$ pump function so any disturbance in the serum Mg2 levels in patients with severe low potassium level should replace with $\mathrm{Mg} 2+$ in spite of the serum $\mathrm{Mg} 2+$ being normal [19].

\section{CONCLUSION}

Our study showed that electrolyte imbalances are the usual problem. Hyponatremia and hypokalemia are the main electrolyte disturbances in both ischemic and hemorrhagic stroke. Patients with normal electrolyte balance had best outcome and it was statistically significant; thus, serum electrolyte assessment should be performed for all stroke patients. The early diagnosis and perfect control and treatment can end with the very good outcome of stroke patients.

\section{AUTHORS' CONTRIBUTIONS}

Aqeel Raheem Hassan: Study design, data interpretation, writing the manuscript, and publication. Zahraa Adel Aryan: Sample collection, writing the manuscript, statistical analysis, and data interpretation.

\section{CONFLICTS OF INTEREST}

There are no conflicts of interest.

\section{REFERENCES}

1. Goldstein LB, Adams R, Alberts MJ, Appel LJ, Brass LM, Bushnell CD, et al. Primary prevention of ischemic stroke: A guideline from the American heart association/American stroke association stroke council: Cosponsored by the atherosclerotic peripheral vascular disease interdisciplinary working group; cardiovascular nursing council; clinical cardiology council; nutrition, physical activity, and metabolism council; and the quality of care and outcomes research interdisciplinary working group: The American academy of neurology affirms the value of this guideline. Stroke 2016;37:1583-633.

2. Broderick J, Connolly S, Feldmann E, Hanley D, Kase C, Krieger D, et al. Guidelines for the management of spontaneous intracerebral hemorrhage in adults 2007 update. Stroke 2007;38:2001-23. 
3. Hankey GJ. Potential new risk factors for ischemic stroke. What is their potential? Stroke 2006;37:2181-8.

4. National Institutes of Health. Adult treatment panel III: Detection, Evaluation, and Treatment of High Blood Cholesterol in Adults. Bethesda, MD. National Institutes of Health; 2002.

5. Megherbi SE, Milan C, Minier D, Couvreur G, Osseby GV, Tilling K, et al. Association between diabetes and stroke subtype on survival and functional outcome 3 months after stroke: Data from the European BIOMED stroke project. Stroke 2003;34:688-94.

6. Wang GQ, Sun W, Wells GA, Li Z, Li T, Wu J. Baseline study on a stroke-related cohort in the rural community of Shanghai. Zhonghua Liu Xing Bing Xue Za Zhi 2006;27:12-4.

7. Tseng CH, Chong CK, Sheu JJ, Wu TH, Tseng CP. Prevalence and risk factors for stroke in Type 2 diabetic patients in Taiwan: A crosssectional survey of a national sample by telephone interview. Diabetic medicine: J Br Diabet Assoc 2005;22:477-82.

8. Al-Rubeaan K, Youssef AM, Subhani SN, Ahmad NA, Meves SH, Diehm $\mathrm{C}$, et al. Ischemic stroke and its risk factors in a registry-based large cross-sectional diabetic cohort in a country facing a diabetes epidemic. J Diabetes Res 2016;4:132-589.

9. Palm F, Urbanek C, Wolf J, Buggle F, Kleemann T, Hennerici MG. Etiology, risk factors and sex differences in ischemic stroke in the Ludwigshafen stroke study, a population-based stroke registry. Cerebrovasc Dis (Basel, Switzerland) 2012;33:69-75.

10. Geddes JM, Fear J, Tennant A, Pickering A, Hillman M, Chamberlain MA, et al. Prevalence of self-reported stroke in a population in northern England. J Epidemiol Community Health 1996;50:140-3.

11. Mozaffarian D, Mussolino ME, Nasir K, Neumar RW, Palaniappan L, Benjamin EJ, et al. Heart disease and stroke statistics-2016 update: A report from the American heart association. Circulation 2016;133:e38-60.

12. Orlandi G, Gelli A, Fanucchi S, Tognoni G, Acerbi G, Murri L. Prevalence of stroke and transient ischaemic attack in the elderly population of an Italian rural community. Eur J Epidemiolo 2003;18:879-82.

13. Boix R, del Barrio JL, Acosta J, Bergareche A, Bermejo-Pareja F,
Gabriel R, et al. Stroke prevalence among the Spanish elderly: An analysis based on screening surveys. BMC Neurol 2006;6:36.

14. Alam MN, Uddin MJ, Rahman KM, Ahmed S, Akhtar M, Nahar N, et al. Electrolyte changes in stroke. Mymensingh Med J 2012;21:594-9.

15. Kusuda K, Saku Y, Sadoshima S, Kozo I, Fujishima M. Disturbances of fluid and electrolyte balance in patients with acute stroke. Nihon Ronen Igakkai Zasshi 2011;26:223-7.

16. Qureshi AI, Suri MF, Sung GY, Straw RN, Yahia AM, Saad M, et al. Prognostic significance of hypernatremia and hyponatremia among patients with aneurysmal subarachnoid hemorrhage. Neurosurgery 2002;50:749-55.

17. Chandy D, Sy R, Aronow WS, Lee WN, Maguire G, Murali R. Hyponatremia and cerebrovascular spasm in aneurysmal subarachnoid hemorrhage. Neurol India 2006;54:273-5.

18. Sherlock M, O'Sullivan E, Agha A, Behan LA, Rawluk D, Brennan P, et al. The incidence and pathophysiology of hyponatremia after subarachnoid hemorrhage. Clin Endocrinol (Oxf) 2012;64:250-4.

19. McGirt MJ, Blessing R, Nimjee SM, Friedman AH, Alexander MJ, Laskowitz DT, et al. Correlation of serum brain natriuretic peptide with hyponatremia and delayed ischemic neurological deficits after subarachnoid hemorrhage. Neurosurgery 2004;54:1369-73.

20. Cheng CJ, Kuo E, Huang CL. Extracellular potassium homeostasis: Insights from hypokalemic periodic paralysis. Semin Nephrol 2013;33:237-47.

21. Gariballa SE, Robinson TG, Fotherby MD. Hypokalemia and potassium excretion in stroke patients. J Am Geriatr Soc 1997;45:1454-58.

22. Fofi L, Dall'armi V, Durastanti L, Valenza A, Lorenzano S, Prencipe M. An observational study on electrolyte disorders in the acute phase of ischemic stroke and their prognostic value. J Clin Neurosci 2012;19:513-6.

23. Hidayah K, Zullies I, Abdul G. Adherence to secondary stroke prevention therapies in ischemic stroke patients at teaching hospital in Central Java Indonesia. Asian J Pharm Clin Res 2017;5:28-30.

24. Ketut W, Putri LA, Eka P. The aspect of neurocognitive and rehabilitation on alexia without agraphia case. Asian J Pharm Clin Res 2018;11:4-6. 